\title{
Unlocking the True Capability of Graphite-based Dual-Ion Batteries with Ethyl Methyl Carbonate Electrolyte
}

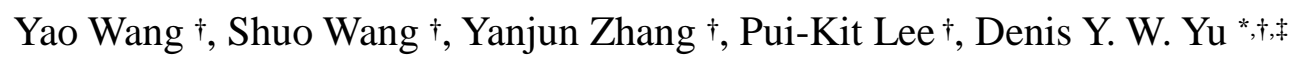

$\dagger$ School of Energy and Environment, City University of Hong Kong, Tat Chee Avenue, Kowloon, Hong Kong SAR. denisyu@cityu.edu.hk

$\$$ Center of Super-Diamond and Advanced Films (COSDAF), City University of Hong Kong, Tat Chee Avenue, Kowloon, Hong Kong SAR.

Email: denisyu@ cityu.edu.hk 

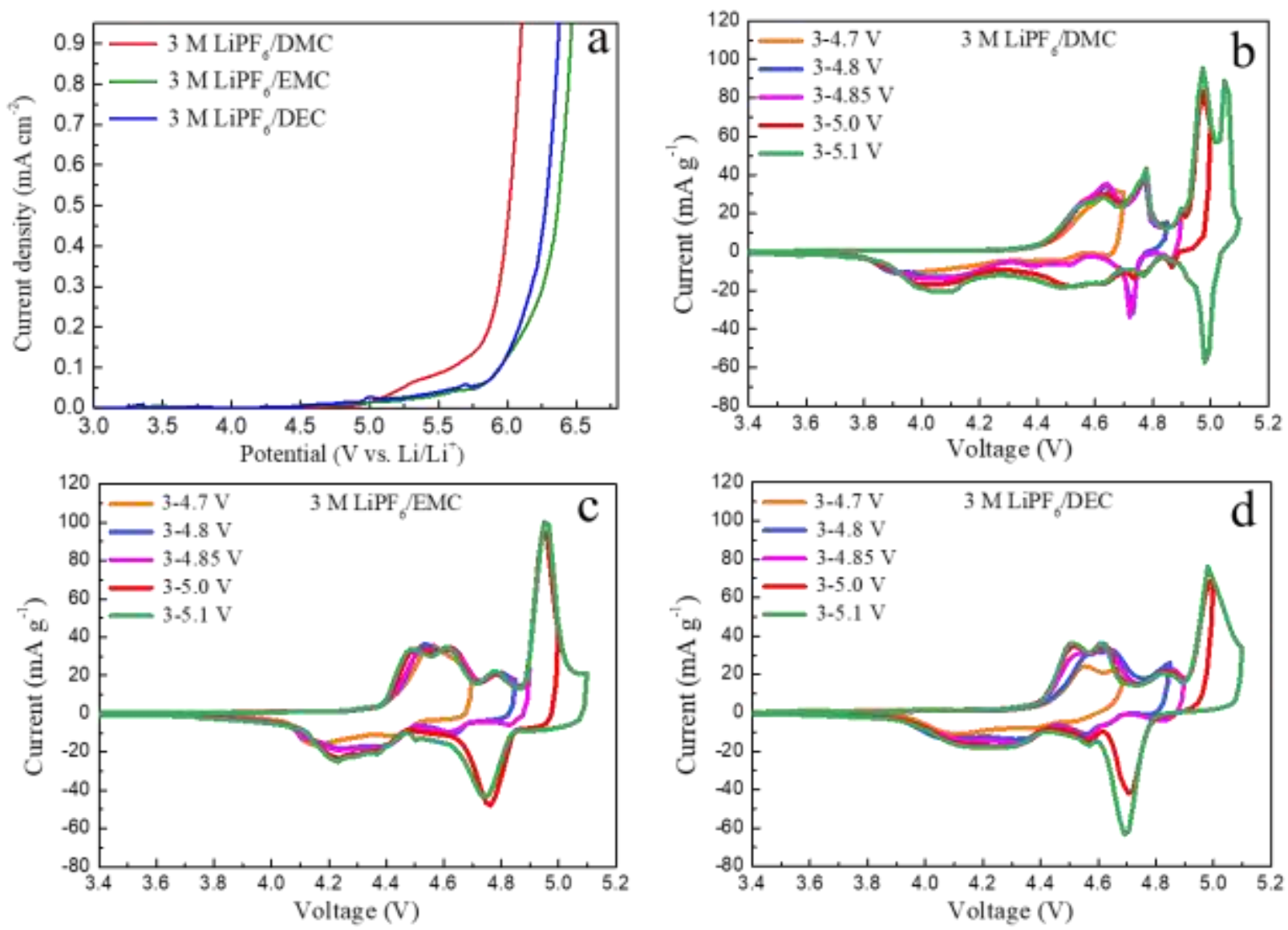

Supplementary Fig. S1 (a) LSV measurements of a C-coated aluminum foil with a scan rate of $1 \mathrm{mV}$ $\mathrm{s}^{-1}$. CV curves of the KS6/Li half cells cycled to different upper voltage in $3 \mathrm{M} \mathrm{LiPF}_{6}$-based (b) DMC, (c) EMC and (d) DEC electrolytes. 

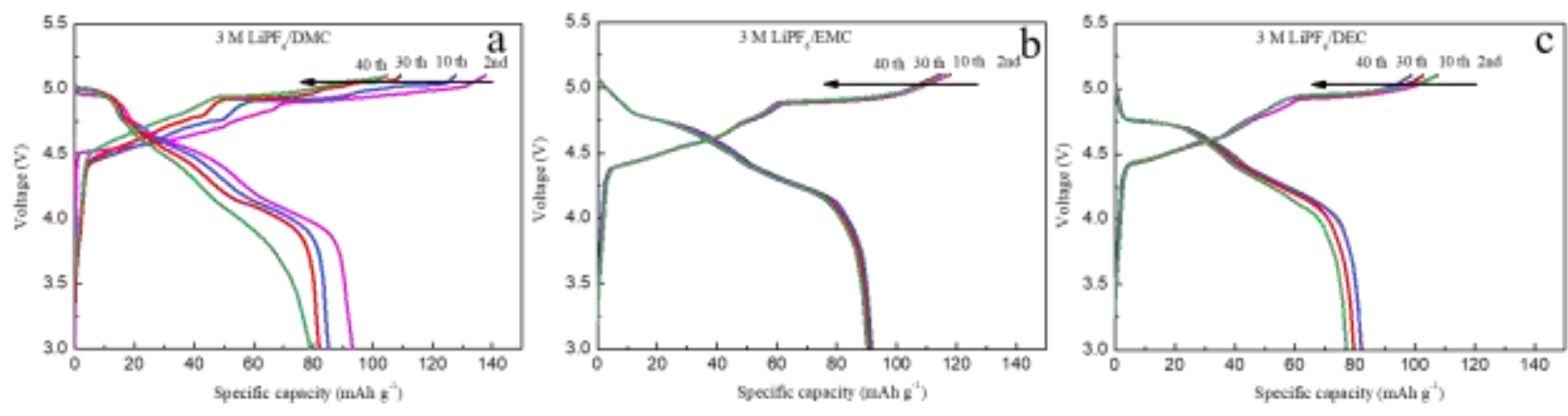

Supplementary Fig. S2 Charge-discharge curves of KS6/Li half cells at different cycles using $3 \mathrm{M}$

$\mathrm{LiPF}_{6}$ based (a) DMC, (b) EMC and (c) DEC electrolytes at $10 \mathrm{~mA} \mathrm{~g}^{-1}$. 


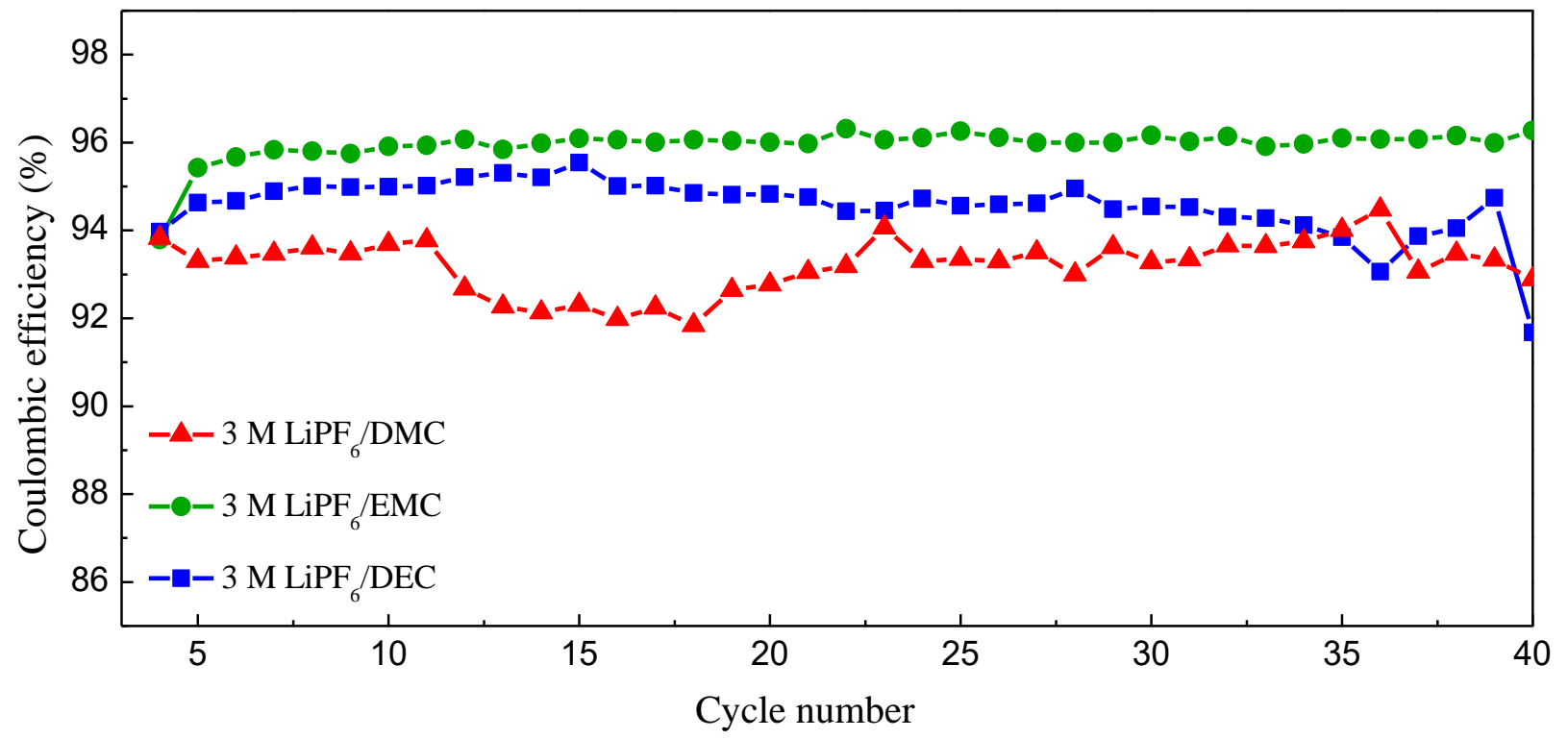

Supplementary Fig. S3 Coulombic efficiency of KS6/Li half cells with 3 M LiPF6-based electrolytes at $100 \mathrm{~mA} \mathrm{~g}^{-1}$. Cutoff voltage: 3-5.1 V. 

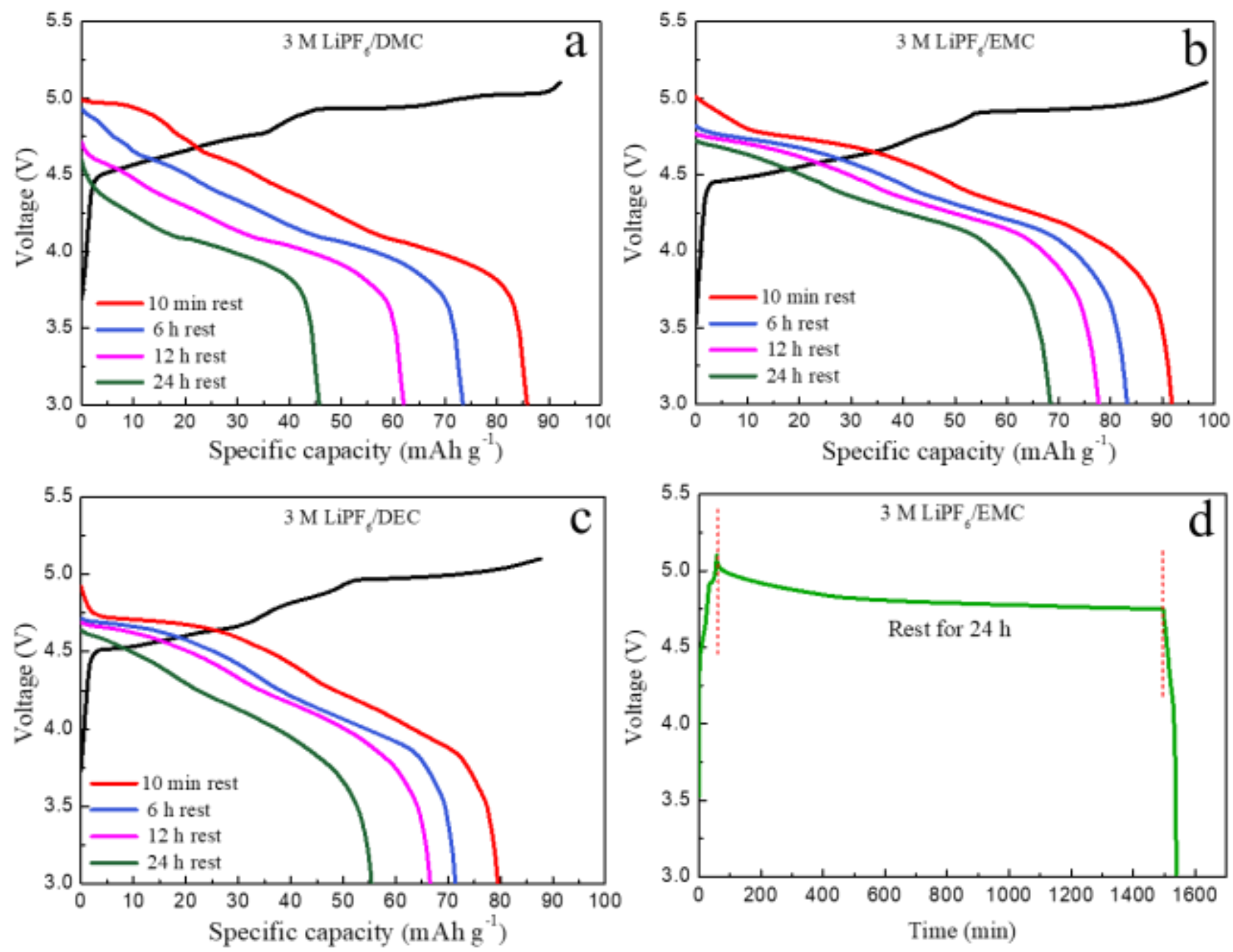

Supplementary Fig. S4 Charge/discharge curves of the KS6 cathodes with 3 M LiPF 6 based (a) DMC,

(b) EMC and (c) DEC electrolytes after rest for 6, 12, 24 hours. (d) the voltage-time curve for resting 24 h with $3 \mathrm{M} \mathrm{LiPF}_{6} / \mathrm{EMC}$ electrolyte.

Fig. S4d shows the voltage profiles of the KS6/Li cell with EMC electrolyte that was charged to $5.1 \mathrm{~V}$ and rested for $24 \mathrm{~h}$. After 24 hours rest, it is observed that the cell voltage decreased from 5.1 $\mathrm{V}$ to $4.75 \mathrm{~V}$ due to the self-discharge, corresponding to a voltage decay rate of $0.014 \mathrm{~V} \mathrm{~h}^{-1}$. In literature, Winter et al. found the graphite/Li dual ion cell gives a voltage decay rate of $0.013 \mathrm{~V} \mathrm{~h}^{-1}$ during $24 \mathrm{~h}$ rest at $20{ }^{\circ} \mathrm{C}$ using $\operatorname{Pyr}_{14}$ TFSI ionic liquid. ${ }^{1}$ However, recently, a higher voltage decay rate of $0.048 \mathrm{~V} \mathrm{~h}^{-1}$ after resting for $25 \mathrm{~h}$ was obtained from the graphite/PCT DIBs with the same ionic liquid electrolyte, as discussed recently by Yuan et al. Thus, the EMC-based electrolyte can give a low self-discharge rate comparable to ionic liquid electrolytes. ${ }^{2}$ 

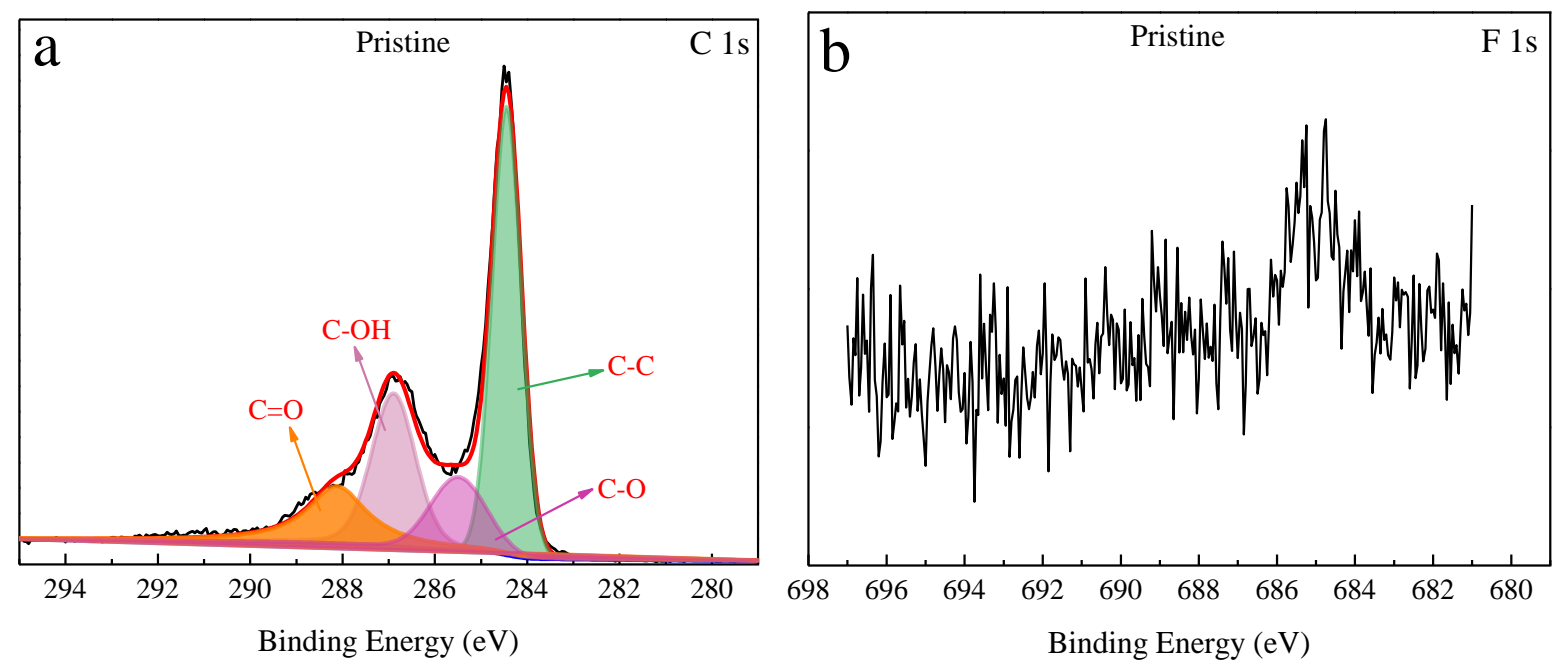

Supplementary Fig. S5 XPS curve fittings for the C 1s, F 1s peaks of the pristine KS6 electrode. 

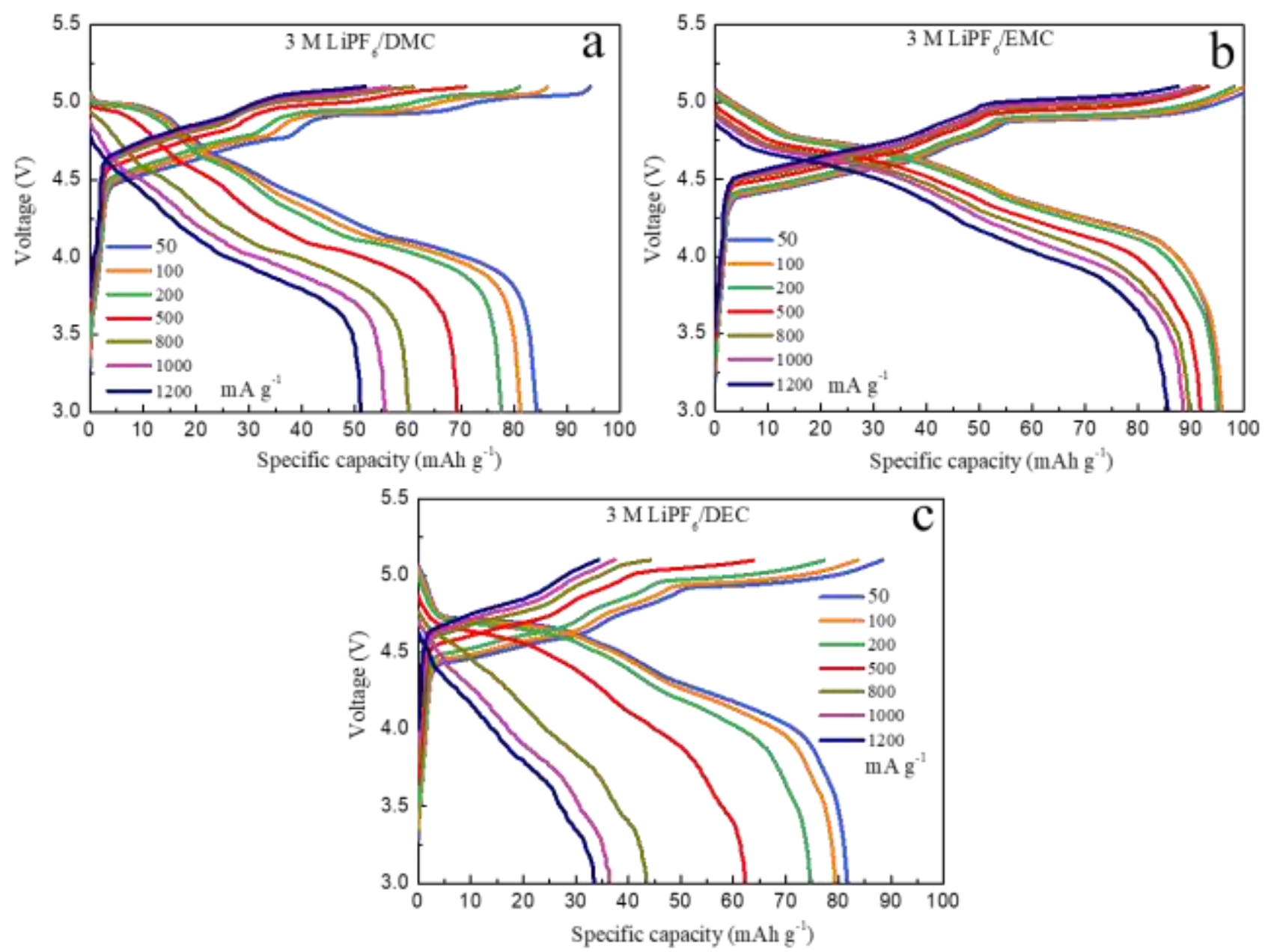

Supplementary Fig. S6 Charge/discharge curves of the KS6 cathodes with 3 M LiPF 6 based (a) DMC,

(b) EMC and (c) DEC electrolytes at various current rates of 50, 100, 200, 500, 800, 1000 and 1200 $\mathrm{mA} \mathrm{g}^{-1}$. 


\section{References}

1. Placke, T.; Fromm, O.; Lux, S. F.; Bieker, P.; Rothermel, S.; Meyer, H. W.; Passerini, S.; Winter, M. Reversible Intercalation of Bis(trifluoromethanesulfonyl)imide Anions from an Ionic Liquid Electrolyte into Graphite for High Performance Dual-Ion Cells. J. Electrochem. Soc. 2012, 159, A1755-A1765.

2. Fang, Y.; Chen, C.; Fan, J.; Zhang, M.; Yuan, W.; Li, L., Reversible Interaction of 1-butyl-1-methylpyrrolidinium Cations with 5,7,12,14-pentacenetetrone from a Pure Ionic Liquid Electrolyte for Dual-Ion Batteries, Chem. Commun. 2019, 55, 8333-8336. 